hep-ph/0410370

February 2, 2008

\title{
Symmetries of the Standard Model
}

\author{
Scott Willenbrock \\ Department of Physics, University of Illinois at Urbana-Champaign \\ 1110 West Green Street, Urbana, IL 61801
}

\begin{abstract}
I present an overview of the standard model, concentrating on its global continuous symmetries, both exact and approximate. There are four lectures, dedicated to spacetime symmetry, flavor symmetry, custodial symmetry, and scale symmetry. Topics include Weyl, Majorana, and Dirac spinors; massive neutrinos; electroweak symmetry breaking; effective field theory; and the hierarchy problem.
\end{abstract}


I was asked to give four lectures entitled Introduction to the Standard Model. However, I suspect all TASI students already have some familiarity with the standard model, so I do not intend to start from scratch. Instead, I would like to talk about some advanced topics within the standard model, ones that are not necessarily covered in a first course. After I finished preparing these lectures, I noticed that there was an underlying theme of symmetry, hence the title of these lectures.

The first two lectures are aimed at understanding neutrinos. Neutrino mass is definitely physics beyond the standard model. My aim is to convince you of this. The first lecture is a general introduction to fermion fields, in particular their transformation properties under spacetime symmetry. The second lecture is concerned with flavor symmetry in both the quark and lepton sector.

The last two lectures are about the Higgs sector of the standard model. Lecture 3 discusses custodial symmetry both within the standard model and more generally. Lecture 4 discusses why the standard Higgs model is problematic when you embed it in a more fundamental theory characterized by a very high energy scale, such as in grand unification.

Although the history of particle physics is fascinating, my presentation will not follow the historical development of these topics. Instead, I will try to present things in a logical manner, deriving as many things as I can from scratch. Some of the details I leave to you in the form of exercises; solutions are included in an Appendix. Occasionally I will make a leap, but I will try to point you to places where you can fill in the gap.

\section{Weyl, Majorana, Dirac}

We all hear (and use) the names Weyl, Majorana, and Dirac in the context of fermions, but there tends to be some confusion about them. In this lecture I hope to clarify their use. I will discuss the Lorentz-transformation properties of fermions, but I will stop short of actually quantizing the fields in terms of creation and annihilation operators. Thus I will only be dealing with the classical aspects of these fields. The quantized fermion field is dealt with in many textbooks. I will usually work with the notation and conventions of Peskin and Schroeder [1], which is an excellent introduction to quantum field theory.

The generators of the Lorentz group are the rotations, $J_{i}$, and the boosts, $K_{i}$. They satisfy the algebra [2]

$$
\begin{aligned}
& {\left[J_{i}, J_{j}\right]=i \epsilon_{i j k} J_{k}} \\
& {\left[K_{i}, K_{j}\right]=-i \epsilon_{i j k} J_{k}} \\
& {\left[J_{i}, K_{j}\right]=i \epsilon_{i j k} K_{k} .}
\end{aligned}
$$

The $J_{i}$ are Hermitian, and the $K_{i}$ are anti-Hermitian. The $J_{i}$ satisfy the algebra of the rotation group, $S U(2)$. The last commutation relation expresses the fact that a boost transforms as a three-vector under rotations. To disentangle the algebra, define the Hermitian generators

$$
\begin{aligned}
A_{i} & =\frac{1}{2}\left(J_{i}+i K_{i}\right) \\
B_{i} & =\frac{1}{2}\left(J_{i}-i K_{i}\right) .
\end{aligned}
$$


Exercise 1.1 - Show that the $A_{i}$ and $B_{i}$ satisfy the algebra

$$
\begin{aligned}
& {\left[A_{i}, A_{j}\right]=i \epsilon_{i j k} A_{k}} \\
& {\left[B_{i}, B_{j}\right]=i \epsilon_{i j k} B_{k}} \\
& {\left[A_{i}, B_{j}\right]=0}
\end{aligned}
$$

The algebra for the $A_{i}$ and the $B_{i}$ is that of $S U(2)$, and the two algebras are independent. We have thus shown that the Lorentz group, $S O(3,1)$, is locally isomorphic to $S U(2) \times S U(2)$.

Representations of $S U(2)$ are familiar from the representations of the rotation group: each representation is label by "spin," which can have integer or half-integer values. Thus the representations of the Lorentz group are labeled $(a, b)$, where $a, b=1 / 2,1,3 / 2, \ldots$. The simplest representation is $(0,0)$, which corresponds to a scalar field. The simplest nontrivial representation is $(1 / 2,0)$, which corresponds to a Weyl spinor, $\chi$. The generators are

$$
\begin{aligned}
A_{i} & =\frac{1}{2} \sigma_{i} \\
B_{i} & =0
\end{aligned}
$$

which corresponds to

$$
\begin{aligned}
J_{i} & =\frac{1}{2} \sigma_{i} \\
i K_{i} & =\frac{1}{2} \sigma_{i} .
\end{aligned}
$$

Hence a Weyl spinor is a 2-component object that transforms under rotations and boosts as

$$
\begin{aligned}
& \chi \rightarrow e^{-\frac{i}{2} \sigma \cdot \theta} \chi \\
& \chi \rightarrow e^{-\frac{1}{2} \sigma \cdot \eta} \chi
\end{aligned}
$$

where $\eta$ is the rapidity, which is related to the velocity by $\beta=\tanh \eta$. Here I am taking the "active" viewpoint, transforming the spinor while keeping the coordinate system fixed. The transformation under rotations shows that a Weyl spinor carries spin 1/2.

Exercise 1.2 - Calculate the transformations under rotations and boosts of the $(0,1 / 2)$ representation.

Let us construct a Lorentz-invariant mass term from a single Weyl spinor. This is called a Majorana mass. It is given by

$$
\mathcal{L}=\frac{1}{2} m\left(\chi^{T} \epsilon \chi+\text { h.c. }\right)
$$

where $\epsilon \equiv i \sigma_{2}$ is the $2 \times 2$ antisymmetric matrix. Let's show that this mass term is Lorentz invariant. Denote a Lorentz transformation acting on $\chi$ by the matrix $M$, where

$$
M=e^{-\frac{i}{2} \sigma \cdot \theta} \text { or } e^{-\frac{1}{2} \sigma \cdot \eta}
$$


Thus under a Lorentz transformation,

$$
\chi^{T} \epsilon \chi \rightarrow \chi^{T} M^{T} \epsilon M \chi
$$

Displaying indices,

$$
\left(M^{T}\right)_{\alpha \beta} \epsilon_{\beta \gamma} M_{\gamma \delta}=\epsilon_{\beta \gamma} M_{\beta \alpha} M_{\gamma \delta}=\epsilon_{\alpha \delta} \operatorname{det} M=\epsilon_{\alpha \delta}
$$

where the last step uses the fact that $\operatorname{det} M=1$. Thus $M^{T} \epsilon M=\epsilon$, which completes the proof in Eq. (4) that $\chi^{T} \epsilon \chi$ is Lorentz invariant. Incidentally, this also shows that the Lorentz group is locally isomorphic to $S L(2, \mathcal{C})$, the group of $2 \times 2$ complex matrices of unit determinant 3 .

Although a Majorana mass is less familiar than a Dirac mass, it is actually a more basic quantity, constructed from a single Weyl spinor. In this sense a Majorana mass is the simplest fermion mass term. However, if $\chi$ carries an unbroken global or local $U(1)$ charge, a Majorana mass is forbidden, since it would violate this symmetry. Thus none of the fermions of the standard model (except neutrinos) can have a Majorana mass, since they carry electric charge. More generally, if $\chi$ transforms under a complex or pseudoreal representation of an unbroken global or local internal symmetry, a Majorana mass is forbidden. Let

$$
\chi \rightarrow U \chi
$$

where $U$ is a unitary transformation acting on a set of Weyl spinors. The mass term in Eq. (4) transforms as

$$
\chi^{T} \epsilon \chi \rightarrow \chi^{T} U^{T} \epsilon U \chi=\chi^{T} \epsilon U^{T} U \chi
$$

where in the last step I have used the fact that $U$ and $\epsilon$ act on different spaces. This is invariant only if $U^{T} U=1$, which is true only if the unitary transformation $U$ is real $\left(U^{*}=U\right)[1]$.

Physically, a fermion with a Majorana mass is its own antiparticle [4. It is referred to as a Majorana fermion. It cannot carry an unbroken global or local $U(1)$ charge (or, more generally, transform under a complex or pseudoreal representation) because a particle and an antiparticle must carry opposite charge.

Exercise 1.3 - Gluinos are hypothetical Majorana fermions that are the superpartners of gluons. Why can they carry color charge?

Exercise 1.4 - Consider a Weyl fermion that transforms under the defining representation of an unbroken $S U(2)$ group. Show that the Majorana mass term

$$
\mathcal{L}=\frac{1}{2} m\left(\epsilon_{a b} \chi^{a T} \epsilon \chi^{b}+\text { h.c. }\right)
$$

is invariant under the $S U(2)$ symmetry. However, show that this term vanishes.

If a Weyl fermion transforms under a complex or pseudoreal representation of an unbroken global or local symmetry, then we need to introduce a second Weyl fermion that transforms under the complex-conjugate representation in order to construct a mass term. This is a 
Dirac mass. Let $\chi, \xi$ transform under the $(1 / 2,0)$ representation of the Lorentz group, and transform under some unbroken global or local symmetry as

$$
\begin{aligned}
& \chi \rightarrow U \chi \\
& \xi \rightarrow U^{*} \xi
\end{aligned}
$$

Then a Lorentz-invariant mass term may be formed which respects the unbroken symmetry,

$$
\mathcal{L}=m\left(\xi^{T} \epsilon \chi+\text { h.c. }\right)
$$

since

$$
\xi^{T} \epsilon \chi \rightarrow \xi^{T} U^{\dagger} \epsilon U \chi=\xi^{T} \epsilon \chi .
$$

Thus it takes two Weyl spinors to construct a Dirac mass. A fermion with a Dirac mass is called a Dirac fermion.

That's pretty much all the physics in this lecture; the rest is mathematics, in order to make contact with the standard way of treating this subject. However, there is a result at the end of the lecture that will be of physical interest in Lecture 2.

Thus far we have constructed a Majorana mass from a Weyl spinor, and a Dirac mass from a pair of Weyl spinors. Let us now introduce a new object, the Dirac spinor, which is a four-component object constructed from a pair of $(1 / 2,0)$ Weyl spinors $\chi, \xi$ via

$$
\psi=\left(\begin{array}{c}
\chi \\
\epsilon \xi^{*}
\end{array}\right)
$$

In terms of a Dirac spinor, a Dirac mass is written in the familiar form

$$
\begin{aligned}
\mathcal{L}=-m \bar{\psi} \psi & =-m\left(\chi^{\dagger},-\xi^{T} \epsilon\right)\left(\begin{array}{ll}
0 & 1 \\
1 & 0
\end{array}\right)\left(\begin{array}{c}
\chi \\
\epsilon \xi^{*}
\end{array}\right) \\
& =m\left(\xi^{T} \epsilon \chi-\chi^{\dagger} \epsilon \xi^{*}\right)
\end{aligned}
$$

where the final expression is identical with Eq. (9). We are using a specific basis for the Dirac gamma matrices, the so-called Weyl or chiral basis,

$$
\gamma^{0}=\left(\begin{array}{ll}
0 & 1 \\
1 & 0
\end{array}\right) \quad \gamma^{i}=\left(\begin{array}{cc}
0 & \sigma^{i} \\
-\sigma^{i} & 0
\end{array}\right) \quad \gamma_{5}=\left(\begin{array}{cc}
-1 & 0 \\
0 & 1
\end{array}\right),
$$

where each entry in the above matrices is itself a $2 \times 2$ matrix. In this basis, the chiral projection operator $\left(1 \pm \gamma_{5}\right) / 2$ projects out the Weyl spinors,

$$
\psi=\frac{1-\gamma_{5}}{2} \psi+\frac{1+\gamma_{5}}{2}=\psi_{L}+\psi_{R}
$$

where

$$
\begin{aligned}
& \psi_{L}=\left(\begin{array}{c}
\chi \\
0
\end{array}\right) \\
& \psi_{R}=\left(\begin{array}{c}
0 \\
\epsilon \xi^{*}
\end{array}\right) .
\end{aligned}
$$


Thus $\psi_{L}$ is the four-component Dirac-spinor version of the Weyl spinor $\chi$, and similarly for $\psi_{R}$ and $\epsilon \xi^{*}$.

Exercise 1.5 - If $\xi$ transforms under the $(1 / 2,0)$ representation of the Lorentz group, show that $\epsilon \xi^{*}$ transforms under the $(0,1 / 2)$ representation. [Hint: recall $M^{T} \epsilon M=\epsilon$, which we derived in Eq. (5)].

Thus a Dirac spinor transforms under the $(1 / 2,0) \oplus(0,1 / 2)$ representation of the Lorentz group, corresponding to $\psi=\psi_{L}+\psi_{R}$.

While a Dirac spinor is composed of two Weyl spinors, a Majorana spinor is a fourcomponent object composed of a single Weyl spinor,

$$
\psi_{M}=\left(\begin{array}{c}
\chi \\
\epsilon \chi^{*}
\end{array}\right) .
$$

Thus it is simply a four-component version of a Weyl spinor.

Exercise 1.6 - Show that

$$
\mathcal{L}=-\frac{1}{2} m \bar{\psi}_{M} \psi_{M}
$$

is a Majorana mass term.

We can find even more ways of writing fermion mass terms by introducing the chargeconjugation matrix $C$, which in the Weyl or chiral representation of the Dirac matrices is

$$
C=\left(\begin{array}{cc}
-\epsilon & 0 \\
0 & \epsilon
\end{array}\right) \text {. }
$$

Given a Dirac spinor $\psi$, we can form the conjugate spinor via

$$
\begin{aligned}
\psi^{c} & \equiv C \gamma^{0} \psi^{*} \\
& =\left(\begin{array}{cc}
-\epsilon & 0 \\
0 & \epsilon
\end{array}\right)\left(\begin{array}{ll}
0 & 1 \\
1 & 0
\end{array}\right)\left(\begin{array}{c}
\chi^{*} \\
\epsilon \xi
\end{array}\right) \\
& =\left(\begin{array}{c}
\xi \\
\epsilon \chi^{*}
\end{array}\right) .
\end{aligned}
$$

Thus

$$
\begin{aligned}
\psi_{L} & =\left(\begin{array}{c}
\chi \\
0
\end{array}\right) \\
\left(\psi^{c}\right)_{L} & =\left(\begin{array}{l}
\xi \\
0
\end{array}\right)=\left(\psi_{R}\right)^{c} .
\end{aligned}
$$

Note that the last relation implies that conjugation and chiral projection do not commute. Exercise 1.7 - Show that

$$
\mathcal{L}=-m\left(\left(\psi^{c}\right)_{L}^{T} C \psi_{L}+\text { h.c. }\right)
$$

is a Dirac mass. 
Exercise 1.8 - Show that

$$
\mathcal{L}=-\frac{1}{2} m\left(\psi_{L}^{T} C \psi_{L}+\text { h.c. }\right)
$$

is a Majorana mass. Thus one can write a Majorana mass in terms of a Dirac spinor.

Exercise 1.9 - Show that

$$
\psi_{M}^{c}=\psi_{M}
$$

This is called the Majorana condition.

In Exercise 1.8 we wrote a Majorana mass in terms of a Dirac spinor. Can we write a Dirac mass in terms of Majorana spinors? Yes, and this is the physically relevant result I promised you earlier in the lecture. Consider a Dirac mass written in terms of a Dirac spinor,

$$
\mathcal{L}=-m \bar{\psi} \psi=-\frac{1}{2} m\left(\bar{\psi} \psi+\bar{\psi}^{c} \psi^{c}\right)
$$

where I'll let you verify the last equality. Now define the Majorana spinors

$$
\begin{aligned}
\psi_{M}^{1} & \equiv \frac{1}{\sqrt{2}}\left(\psi+\psi^{c}\right) \\
\psi_{M}^{2} & \equiv \frac{1}{\sqrt{2}}\left(\psi-\psi^{c}\right) .
\end{aligned}
$$

Then Eq. (20) can be written

$$
\mathcal{L}=-\frac{1}{2} m\left(\bar{\psi}_{M}^{1} \psi_{M}^{1}+\bar{\psi}_{M}^{2} \psi_{M}^{2}\right)
$$

Thus a Dirac fermion is equivalent to two degenerate Majorana fermions. However

$$
\begin{aligned}
\left(\psi_{M}^{1}\right)^{c} & =\frac{1}{\sqrt{2}}\left(\psi^{c}+\psi\right)=\psi_{M}^{1} \\
\left(\psi_{M}^{2}\right)^{c} & =\frac{1}{\sqrt{2}}\left(\psi^{c}-\psi\right)=-\psi_{M}^{2} .
\end{aligned}
$$

Thus the two Majorana spinors have the opposite sign under charge conjugation. The Majorana spinor $\psi_{M}^{2}$ is therefore of the form

$$
\psi_{M}^{2}=\left(\begin{array}{c}
\chi \\
-\epsilon \chi^{*}
\end{array}\right)
$$

which is a generalization of the construction in Eq. (14). We will see at the end of Lecture 2 the physical significance of these results.

The various ways we have learned to write Majorana and Dirac masses in terms of Weyl, Majorana, and Dirac spinors are collected in Table 11. As a final exercise, I invite you to derive the one entry in this Table that we have not yet discussed.

Exercise 1.10 - Show that

$$
\mathcal{L}=-\frac{1}{2} m\left({\overline{\left(\psi^{c}\right)}}_{R} \psi_{L}+\text { h.c. }\right)
$$

is a Majorana mass.

This form for the Majorana mass in terms of Dirac spinors is used quite commonly [4. 


\begin{tabular}{|c||c|c|}
\hline spinor & Majorana mass & Dirac mass \\
\hline Weyl & $\frac{1}{2} m\left(\chi^{T} \epsilon \chi+h . c.\right)$ & $m\left(\xi^{T} \epsilon \chi+h . c.\right)$ \\
Majorana & $-\frac{1}{2} m \bar{\psi}_{M} \psi_{M}$ & $-\frac{1}{2} m\left(\bar{\psi}_{M}^{1} \psi_{M}^{1}+\bar{\psi}_{M}^{2} \psi_{M}^{2}\right)$ \\
Dirac & $-\frac{1}{2} m\left(\bar{\psi}_{L}^{T} C \psi_{L}+\right.$ h.c. $)$ & $-m\left(\left(\psi^{c}\right)_{L}^{T} C \psi_{L}+\right.$ h.c. $)$ \\
Dirac & $-\frac{1}{2} m\left({\overline{\left(\psi^{c}\right)_{R}}}_{L}+\right.$ h.c. $)$ & $-m \bar{\psi} \psi$ \\
\hline \hline
\end{tabular}

Table 1: A Majorana mass and a Dirac mass may be constructed from Weyl, Majorana, or Dirac spinors.

\section{Flavor symmetry}

In Table 2 I list the fermion fields that make up the standard model, along with their $S U(3) \times S U(2)_{L} \times U(1)_{Y}$ quantum numbers. The index $i=1,2,3$ on each field refers to the generation. I list the fields in terms of left-chiral Dirac spinors, which, as we saw in the previous section, is the four-component version of a Weyl spinor. For example, one should think of

$$
\begin{aligned}
u_{L} & =\left(\begin{array}{l}
\chi \\
0
\end{array}\right) \\
\left(u^{c}\right)_{L} & =\left(\begin{array}{l}
\xi \\
0
\end{array}\right)
\end{aligned}
$$

where $\chi, \xi$ are $(1 / 2,0)$ Weyl spinors. A priori $u_{L}$ and $\left(u^{c}\right)_{L}$ are totally independent, despite their names. They are only named thusly because we know they will eventually pair up to make a Dirac spinor

$$
u=\left(\begin{array}{c}
\chi \\
\epsilon \xi^{*}
\end{array}\right)
$$

Working in terms of only left-chiral fields is particularly useful for grand unification, where we attempt to combine fermions into representations of a group that contains the standard model as a subgroup. Since left-chiral fields all transform the same way under the Lorentz group, combining them respects Lorentz invariance.

The Lagrangian of the standard model is the sum of the gauge, matter, Yukawa, and Higgs interactions,

$$
\mathcal{L}_{S M}=\mathcal{L}_{\text {Gauge }}+\mathcal{L}_{\text {Matter }}+\mathcal{L}_{\text {Yukawa }}+\mathcal{L}_{\text {Higgs }} .
$$

The pure gauge interactions contain the kinetic energies of the gauge bosons and their selfinteractions. The "matter" Lagrangian contains the kinetic energy and gauge interactions of the fermion fields,

$$
\mathcal{L}_{\text {Matter }}=i \bar{Q}_{L}^{i} \not D Q_{L}^{i}+i{\overline{\left(u^{c}\right)_{L}^{i}}}_{L} \not D\left(u^{c}\right)_{L}^{i}+i{\overline{\left(d^{c}\right)}}_{L}^{i} \not D\left(d^{c}\right)_{L}^{i}+i \bar{L}_{L}^{i} \not D L_{L}^{i}+i{\overline{\left(e^{c}\right)_{L}^{i}}}_{L} \not D\left(e^{c}\right)_{L}^{i} .
$$

A sum on the index $i$, which represents the generation, is implied in the Lagrangian. To put this into the canonical form in terms of left- and right-chiral fields, we use

$$
\left(\psi^{c}\right)_{L}=C \gamma^{0} \psi_{R}^{*}
$$




$$
\begin{aligned}
& \underline{S U(3)} \underline{S U(2)_{L}} \quad \underline{U(1)_{Y}} \\
& Q_{L}^{i}=\left(\begin{array}{c}
u_{L} \\
d_{L}
\end{array}\right) \quad\left(\begin{array}{c}
c_{L} \\
s_{L}
\end{array}\right) \quad\left(\begin{array}{c}
t_{L} \\
b_{L}
\end{array}\right) \quad 3 \quad 12 \quad \frac{1}{6} \\
& \left(u^{c}\right)_{L}^{i}=\quad \begin{array}{llllll}
\left(u^{c}\right)_{L} & \left(c^{c}\right)_{L} & \left(t^{c}\right)_{L} & \overline{3} & 1 & -\frac{2}{3}
\end{array} \\
& \left(d^{c}\right)_{L}^{i}=\quad \begin{array}{llllll}
\left(d^{c}\right)_{L} & \left(s^{c}\right)_{L} & \left(b^{c}\right)_{L} & \overline{3} & 1 & \frac{1}{3}
\end{array} \\
& L_{L}^{i}=\left(\begin{array}{c}
\nu_{e L} \\
e_{L}
\end{array}\right) \quad\left(\begin{array}{c}
\nu_{\mu L} \\
\mu_{L}
\end{array}\right) \quad\left(\begin{array}{c}
\nu_{\tau L} \\
\tau_{L}
\end{array}\right) \quad 1 \quad \begin{array}{lll}
2 & 2
\end{array} \\
& \left(e^{c}\right)_{L}^{i}=\quad \begin{array}{llllll}
\left(e^{c}\right)_{L} & \left(\mu^{c}\right)_{L} & \left(\tau^{c}\right)_{L} & 1 & 1 & 1
\end{array}
\end{aligned}
$$

Table 2: The fermion fields of the standard model and their gauge quantum numbers.

which yields

$$
\mathcal{L}_{\text {Matter }}=i \bar{Q}_{L}^{i} \not D Q_{L}^{i}+i \bar{u}_{R}^{i} \not D u_{R}^{i}+i \bar{d}_{R}^{i} \not D d_{R}^{i}+i \bar{L}_{L}^{i} \not D L_{L}^{i}+i \bar{e}_{R}^{i} \not D e_{R}^{i}
$$

At this stage, all the fermions are massless. Majorana masses are forbidden by the fact that all fermions carry hypercharge; in addition, some transform under a complex representation of $S U(3)$, and some transform under a pseudoreal representation of $S U(2)_{L}$. Dirac masses are forbidden by the fact that no fermion transforms under the complex-conjugate representation of another fermion.

The absence of fermion masses implies that $\mathcal{L}_{\text {Matter }}$ has a good deal of (accidental) global symmetry,

$$
\begin{aligned}
& Q_{L}^{i} \rightarrow U_{Q_{L}}^{i j} Q_{L}^{j} \\
& u_{R}^{i} \rightarrow U_{u_{R}}^{i j} u_{R}^{j} \\
& d_{R}^{i} \rightarrow U_{d_{R}}^{i j} d_{R}^{j} \\
& L_{L}^{i} \rightarrow U_{L_{L}}^{i j} L_{L}^{j} \\
& e_{R}^{i} \rightarrow U_{e_{R}}^{i j} e_{R}^{j}
\end{aligned}
$$

This symmetry is accidental in the sense that it is not imposed, but rather follows from the fermion content and gauge symmetries of the standard model. Since there are five independent $U(3)$ symmetries, the global flavor symmetry of the matter Lagrangian is $[U(3)]^{5}$.

These global flavor symmetries are violated by the Yukawa couplings of the fermions to the Higgs field (see Table 21),

$$
\mathcal{L}_{Y u k a w a}=-\Gamma_{u}^{i j} \bar{Q}_{L}^{i} \epsilon \phi^{*} u_{R}^{j}-\Gamma_{d}^{i j} \bar{Q}_{L}^{i} \phi d_{R}^{j}-\Gamma_{e}^{i j} \bar{L}_{L}^{i} \phi e_{R}^{j}+\text { h.c. }
$$

where $\Gamma_{u}, \Gamma_{d}, \Gamma_{e}$ are $3 \times 3$ complex matrices in generation space.

Exercise 2.1 - Show that if $\phi$ is an $S U(2)_{L}$ doublet, then so is $\epsilon \phi^{*}$ (see Exercise 1.5). 


$$
\begin{aligned}
& \underline{S U(3)} \quad \underline{S U(2)_{L}} \quad \underline{U(1)_{Y}} \\
& \phi=\left(\begin{array}{c}
\phi^{+} \\
\phi^{0}
\end{array}\right) \quad 1 \quad 2 \quad \frac{1}{2}
\end{aligned}
$$

Table 3: The Higgs field and its gauge quantum numbers.

Only a very small subgroup of $[U(3)]^{5}$ is not violated, corresponding to baryon number

$$
\begin{aligned}
Q_{L}^{i} & \rightarrow e^{i \theta / 3} Q_{L}^{i} \\
u_{R}^{i} & \rightarrow e^{i \theta / 3} u_{R}^{i} \\
d_{R}^{i} & \rightarrow e^{i \theta / 3} d_{R}^{i}
\end{aligned}
$$

and lepton number

$$
\begin{aligned}
L_{L}^{i} & \rightarrow e^{i \phi} L_{L}^{i} \\
e_{R}^{i} & \rightarrow e^{i \phi} e_{R}^{i} .
\end{aligned}
$$

Thus baryon number and lepton number are accidental global symmetries of the standard model (see also Exercise 2.2).

When the Higgs field acquires a vacuum expectation value,

$$
\langle\phi\rangle=\left(\begin{array}{c}
0 \\
v / \sqrt{2}
\end{array}\right)
$$

the fermion fields (except neutrinos) become massive via their Yukawa couplings to the Higgs field, Eq. (29),

$$
\mathcal{L}_{M}=-M_{u}^{i j} \bar{u}_{L}^{i} u_{R}^{j}-M_{d}^{i j} \bar{d}_{L}^{i} d_{R}^{j}-M_{e}^{i j} \bar{e}_{L}^{i} e_{R}^{j}+\text { h.c. },
$$

where

$$
M^{i j}=\Gamma^{i j} \frac{v}{\sqrt{2}}
$$

are fermion mass matrices. Thus $\psi_{L}$ and $\psi_{R}=C \gamma^{0}\left(\psi^{c}\right)_{L}^{*}$ have paired up to make Dirac masses for $u^{i}, d^{i}, e^{i}$. The neutrino field $\nu_{L}$ carries no unbroken gauge symmetry, so it could potentially acquire a Majorana mass

$$
\mathcal{L}=-\frac{1}{2} M_{\nu}^{i j}\left(\nu_{L}^{i T} C \nu_{L}^{j}+\text { h.c. }\right) .
$$

However, this term is forbidden by the accidental lepton number symmetry. Actually, baryon number and lepton number are anomalous, but $B-L$ is not [1. So it is more precise to say that a Majorana neutrino mass is forbidden by $B-L$.

The Yukawa matrices $\Gamma$ in Eq. (29) are $3 \times 3$ complex matrices, and since there are three of them we have apparently introduced $3 \times 3 \times 3 \times 2=54$ new parameters into the theory. However, we will now show that only a subset of these parameters are physically relevant.

Given this Lagrangian, one can proceed to calculate any physical process of interest. However, it is convenient to first perform field redefinitions to make the physical content of 
the theory manifest. These field redefinitions do not change the predictions of the theory; they are analogous to a change of variables when performing an integration. To make the masses of the fermions manifest, we perform unitary field redefinitions on the fields in order to diagonalize the mass matrices in Eq. (31):

$$
\begin{array}{cc}
u_{L}^{i}=A_{u_{L}}^{i j} u_{L}^{\prime j} & u_{R}^{i}=A_{u_{R}}^{i j} u_{R}^{\prime j} \\
d_{L}^{i}=A_{d_{L}}^{i j} d_{L}^{\prime j} & d_{R}^{i}=A_{d_{R}}^{i j} d_{R}^{\prime j} \\
e_{L}^{i}=A_{e_{L}}^{i j} e_{L}^{\prime j} & e_{R}^{i}=A_{e_{R}}^{i j} e_{R}^{\prime j} \\
\nu_{L}^{i}=A_{\nu_{L}}^{i j} \nu_{L}^{\prime j} . &
\end{array}
$$

Each matrix $A$ must be unitary in order to preserve the form of the kinetic-energy terms in the matter Lagrangian, Eq. (28), e.g.

$$
\mathcal{L}_{K E}=i \bar{u}_{L} \not \partial u_{L}=i \bar{u}_{L}^{\prime} A_{u_{L}}^{\dagger} \not \partial A_{u_{L}} u_{L}^{\prime}=i \bar{u}_{L}^{\prime} \not \partial u_{L}^{\prime}
$$

where I have switched to index-free notation. Once the mass matrices are diagonalized, the masses of the fermions are manifest. These transformations also diagonalize the Yukawa matrices $\Gamma$, since they are proportional to the mass matrices [see Eq. (32)]. However, we must consider what effect these field redefinitions have on the rest of the Lagrangian. They have no effect on the pure gauge or Higgs parts of the Lagrangian, which are independent of the fermion fields. They do affect the matter part of the Lagrangian, Eq. (28). However, a subset of these field redefinitions is the global $[U(3)]^{5}$ symmetry of the matter Lagrangian; this subset therefore has no effect.

One can count how many physically relevant parameters remain after the field redefinitions are performed [5]. Let's concentrate on the quark sector. The number of parameters contained in the complex matrices $\Gamma_{u}, \Gamma_{d}$ is $2 \times 3 \times 3 \times 2=36$. The unitary symmetries $U_{Q_{L}}, U_{u_{R}}, U_{d_{R}}$ are a subset of the quark field redefinitions; this subset will not affect the matter part of the Lagrangian. There are $3 \times 3 \times 3$ degrees of freedom in these symmetries (a unitary $N \times N$ matrix has $N^{2}$ free parameters), so the total number of parameters that remain in the full Lagrangian after field redefinitions is

$$
2 \times 3 \times 3 \times 2-(3 \times 3 \times 3-1)=10
$$

where I have subtracted baryon number from the subset of field redefinitions that are symmetries of the matter Lagrangian. Baryon number is a symmetry of the Yukawa Lagrangian, Eq. (29), and hence cannot be used to diagonalize the mass matrices. The ten remaining parameters correspond to the six quark masses and the four parameters of the CabibboKobayashi-Maskawa (CKM) matrix (three mixing angles and one $C P$-violating phase).

Exercise 2.2 - Do the same counting for the lepton sector. Show that it yields just one parameter. Argue that this can be interpreted as $m_{e}, m_{\mu}, m_{\tau}$ and two additional global $U(1)$ symmetries. What are they?

Thus far the neutrinos are massless. But we know that neutrinos have mass. Why not extend the standard model to include the field $N_{R}$ (it will be clear shortly why I resist labeling the field $\nu_{R}$ ), and add the Yukawa interaction

$$
\mathcal{L}_{\text {Yukawa }}=-\Gamma_{\nu}^{i j} \bar{L}_{L}^{i} \epsilon \phi^{*} N_{R}^{j}+\text { h.c. . }
$$


Exercise 2.3 - Show that $N_{R}$ is sterile, that is, carries no gauge quantum numbers, and carries lepton number +1 .

Then when the Higgs field acquires a vacuum expectation value, the neutrinos gain a Dirac mass just like all the other fermions. There are two shortcomings to this proposal:

- There is no explanation of why neutrinos are so much lighter than all the other fermions.

- Since $N_{R}$ is sterile, the gauge symmetry allows a Majorana mass term

$$
\mathcal{L}=-\frac{1}{2} M_{R}^{i j} N_{R}^{i T} C N_{R}^{j}+\text { h.c. . }
$$

With this term present, the fields $\nu_{L}$ and $N_{R}$ do not pair up to make a Dirac fermion. Rather, there are two Majorana neutrinos per generation, which is more than we need to describe nature.

We will later show how the latter shortcoming can be turned into a virtue that addresses the first shortcoming.

The Majorana mass term of Eq. (38) is forbidden if lepton number is imposed as an exact symmetry. In that case, the neutrino would acquire a Dirac mass from the Yukawa interaction of Eq. (37). However, recall that lepton number is an accidental symmetry of the standard model; there is no reason to expect it to be an exact symmetry when we extend the standard model. Even if this scenario were realized in nature, we should still regard it as physics beyond the standard model, because it requires the introduction of two new features: the field $N_{R}$, and the elevation of lepton number from an accidental to an exact symmetry.

Rather than add the field $N_{R}$, let's regard the standard model as a low-energy effective field theory, with a Lagrangian that is an expansion in inverse powers of some large mass $M$ [2, 6],

$$
\mathcal{L}=\mathcal{L}_{S M}+\frac{1}{M} \operatorname{dim} 5+\frac{1}{M^{2}} \operatorname{dim} 6+\cdots
$$

The terms beyond the standard model represent operators of higher and higher dimension, starting with dimension five, which is the least suppressed. Given the field content and gauge symmetries of the standard model, there is only one dimension-five operator,

$$
\mathcal{L}_{5}=\frac{c^{i j}}{M} L_{L}^{i T} \epsilon \phi C \phi^{T} \epsilon L_{L}^{j}+\text { h.c. }
$$

Exercise 2.4 - Show that $\mathcal{L}_{5}$ is $S U(2)_{L} \times U(1)_{Y}$ invariant, and that $c^{i j}$ is a symmetric matrix. Show that $\mathcal{L}_{5}$ violates lepton number.

When the Higgs field acquires a vacuum expectation value, Eq. (30), this yields a Majorana mass for the neutrino,

$$
\mathcal{L}_{M a j}=-\frac{c^{i j}}{2} \frac{v^{2}}{M} \nu_{L}^{i T} C \nu_{L}^{j}+\text { h.c. . }
$$

The neutrino is allowed to acquire a Majorana mass because lepton number is violated by $\mathcal{L}_{5}$. Thus lepton number is only a low-energy accidental symmetry, and is in general violated by higher-dimension operators. 
We see from Eq. (41) that neutrino masses are of order $v^{2} / M$. Thus, if $M \gg v$, neutrino masses are naturally much less than $v$. This is an attractive explanation of why neutrinos are so much lighter than all the other fermions.

Exercise 2.5 - Show that the MNS matrix (the analogue of the CKM matrix in the lepton sector) has six physically relevant parameters. [Note: $c^{i j}$ is a symmetric, complex matrix.]

Let's now return to our discussion of the sterile neutrino, $N_{R}^{i}$, and show how it yields Eq. (41) if the sterile neutrino is made very heavy. Begin with the Lagrangians of Eqs. (37) and (38),

$$
\mathcal{L}=-\bar{L}_{L} \Gamma_{\nu} \epsilon \phi^{*} N_{R}-\frac{1}{2} N_{R}^{T} M_{R} C N_{R}+\text { h.c. },
$$

where I have switched to an index-free notation. If $M_{R}$ is very large, then the field $N_{R}$ is not present at low energy, and we can integrate it out of the theory. At low energy, $N_{R}$ acts like a non-dynamical, classical field, and we can remove it by solving its equation of motion,

$$
\frac{\partial \mathcal{L}}{\partial N_{R}}=0
$$

and plugging it back into Eq. (42). This yields Eq. (41), where

$$
\frac{c}{M}=-\frac{1}{2}\left(\Gamma_{\nu} M_{R}^{-1} \Gamma_{\nu}^{T}\right)^{\dagger} .
$$

Thus we see that the mass $M$ in our effective-field-theory description, Eq. (39), is proportional to the mass $M_{R}$. The effective field theory breaks down when one approaches energy of order the mass of the heavy Majorana neutrino $N_{R}$.

Exercise 2.6 - Derive Eq. (44). [Hint: If you follow the above steps, you'll arrive at the h.c. term of $\mathcal{L}_{5}$.]

I show in Fig. 1 1 the spectrum of neutrino masses of a single generation in the two limits we have just discussed. If $M_{R} \gg v$, then $N_{R}$ is a heavy Majorana neutrino of mass $M_{R}$, and $\nu_{L}$ is a very light Majorana neutrino of mass $\mathcal{O}\left(v^{2} / M_{R}\right)$. In the other extreme, if $M_{R}=0$ (which would be the case if lepton number were an exact symmetry), $\nu_{L}$ and $N_{R}$ pair up to make a Dirac neutrino of mass $\mathcal{O}(v)$. This exemplifies the fact that a Dirac neutrino can be thought of as two degenerate Majorana neutrinos (of opposite charge conjugation), which we showed at the end of the previous section.

A heavy neutrino of mass $M_{R}$ yielding a light neutrino of mass $\mathcal{O}\left(v^{2} / M_{R}\right)$ is called the "seesaw" mechanism: compared with the mass scale $v$, one has gone up and the other has gone down [4. The effective-field-theory description, Eq. (39), can be regarded as a generalization of the see-saw mechanism, one that does not depend on the details of the new physics residing at the scale $M$. 


$$
\begin{array}{ll}
M_{R} \gg v & M_{R}=0 \\
\mathcal{O}\left(M_{R}\right) &
\end{array}
$$
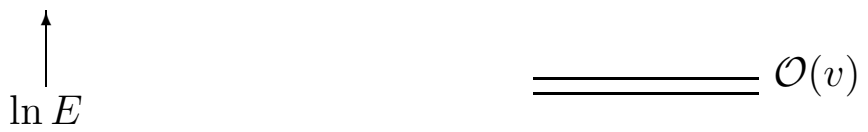

$\mathcal{O}\left(v^{2} / M_{R}\right)$

Figure 1: If $M_{R} \gg v$, there is a heavy Majorana neutrino of mass $\mathcal{O}\left(M_{R}\right)$ and a light Majorana neutrino of mass $\mathcal{O}\left(v^{2} / M_{R}\right)$. If $M_{R}=0$, these neutrinos pair up to make a Dirac neutrino of mass $\mathcal{O}(v)$. 


\section{Custodial symmetry}

We now leave the flavor sector of the standard model to begin a discussion of the Higgs sector, or more generally, the electroweak-symmetry-breaking sector. In this section we consider the global symmetries of this sector.

The Higgs sector of the standard model is described by the Lagrangian

$$
\mathcal{L}_{\text {Higgs }}=\left(D_{\mu} \phi\right)^{\dagger} D^{\mu} \phi-V(\phi)
$$

where the Higgs potential is

$$
V(\phi)=-\mu^{2} \phi^{\dagger} \phi+\lambda\left(\phi^{\dagger} \phi\right)^{2}
$$

and the gauge-covariant derivative is

$$
D_{\mu} \phi=\left(\partial_{\mu}+i \frac{g}{2} \sigma \cdot W_{\mu}+i \frac{g^{\prime}}{2} B_{\mu}\right) \phi .
$$

The Higgs Lagrangian is $S U(2)_{L} \times U(1)_{Y}$ symmetric by construction, but it also has an approximate (accidental) global symmetry. To see this, it is useful to rewrite the Lagrangian as follows. Label the components of the Higgs-doublet field as

$$
\phi=\left(\begin{array}{c}
\phi^{+} \\
\phi^{0}
\end{array}\right)
$$

Then $\epsilon \phi^{*}$, which is also an $S U(2)_{L}$ doublet (see Exercise 2.1), has components

$$
\epsilon \phi^{*}=\left(\begin{array}{c}
\phi^{0 *} \\
-\phi^{-}
\end{array}\right)
$$

where I've used $\phi^{-}=\phi^{+*}$. Now define a Higgs matrix (or bi-doublet) field

$$
\Phi=\frac{1}{\sqrt{2}}\left(\epsilon \phi^{*}, \phi\right)=\frac{1}{\sqrt{2}}\left(\begin{array}{cc}
\phi^{0 *} & \phi^{+} \\
-\phi^{-} & \phi^{0}
\end{array}\right) .
$$

We can rewrite the Higgs Lagrangian in terms of this matrix field as

$$
\mathcal{L}_{\text {Higgs }}=\operatorname{Tr}\left(D_{\mu} \Phi\right)^{\dagger} D^{\mu} \Phi-V(\Phi)
$$

where the potential is given by

$$
V(\Phi)=-\mu^{2} \operatorname{Tr} \Phi^{\dagger} \Phi+\lambda\left(\operatorname{Tr} \Phi^{\dagger} \Phi\right)^{2}
$$

and the gauge-covariant derivative by

$$
D_{\mu} \Phi=\left(\partial_{\mu} \Phi+i \frac{g}{2} \sigma \cdot W_{\mu} \Phi-i \frac{g^{\prime}}{2} B_{\mu} \Phi \sigma_{3}\right) .
$$

Note the Pauli matrix $\sigma_{3}$ at the end of the last term in the above equation. This is necessary because $\phi$ and $\epsilon \phi^{*}$ have opposite hypercharge. 
Exercise 3.1 - Verify that Eqs. (45) and (51) are identical.

The $S U(2)_{L} \times U(1)_{Y}$ gauge symmetry acts on the Higgs matrix field as

$$
\begin{aligned}
S U(2)_{L}: & \Phi \rightarrow L \Phi \\
U(1)_{Y}: & \Phi \rightarrow \Phi e^{-\frac{i}{2} \sigma_{3} \theta}
\end{aligned}
$$

where the $\sigma_{3}$ in the hypercharge transformation is again due to the opposite hypercharges of $\phi$ and $\epsilon \phi^{*}$. Under $S U(2)_{L}$,

$$
\operatorname{Tr}\left(D_{\mu} \Phi\right)^{\dagger} D^{\mu} \Phi \rightarrow \operatorname{Tr}\left(D_{\mu} \Phi\right)^{\dagger} L^{\dagger} L D^{\mu} \Phi=\operatorname{Tr}\left(D_{\mu} \Phi\right)^{\dagger} D^{\mu} \Phi
$$

which shows it is invariant.

To make the approximate global symmetry manifest, take the limit that the hypercharge coupling vanishes, $g^{\prime} \rightarrow 0$. The Higgs Lagrangian is still given by Eq. (51), but now the gauge-covariant derivative is given simply by

$$
D_{\mu} \Phi=\left(\partial_{\mu}+i \frac{g}{2} \sigma \cdot W_{\mu}\right) \Phi .
$$

We see that in this limit the Lagrangian has a global symmetry $S U(2)_{R}$, given by

$$
S U(2)_{R}: \quad \Phi \rightarrow \Phi R^{\dagger} .
$$

Under an $S U(2)_{R}$ transformation,

$$
\operatorname{Tr}\left(D_{\mu} \Phi\right)^{\dagger} D^{\mu} \Phi \rightarrow \operatorname{Tr} R\left(D_{\mu} \Phi\right)^{\dagger} D^{\mu} \Phi R^{\dagger}=\operatorname{Tr}\left(D_{\mu} \Phi\right)^{\dagger} D^{\mu} \Phi
$$

which shows it is invariant. Thus in the limit $g^{\prime} \rightarrow 0$, the Higgs sector of the standard model has the global symmetry $S U(2)_{L} \times S U(2)_{R}$, where $S U(2)_{L}$ is just the global version of the gauge symmetry, and $S U(2)_{R}$ is an approximate, accidental global symmetry:

$$
S U(2)_{L} \times S U(2)_{R}: \quad \Phi \rightarrow L \Phi R^{\dagger} .
$$

Exercise 3.2 - Show that $U(1)_{Y}$ is a subgroup of $S U(2)_{R}$.

When the Higgs field acquires a vacuum expectation value, Eq. (30), the matrix field is

$$
\langle\Phi\rangle=\frac{1}{2}\left(\begin{array}{ll}
v & 0 \\
0 & v
\end{array}\right) .
$$

This breaks both $S U(2)_{L}$ and $S U(2)_{R}$,

$$
L\langle\Phi\rangle \neq\langle\Phi\rangle \quad\langle\Phi\rangle R^{\dagger} \neq\langle\Phi\rangle
$$

but leaves unbroken the subgroup $S U(2)_{L+R}$, corresponding to simultaneous $S U(2)_{L}$ and $S U(2)_{R}$ transformations with $L=R$ :

$$
L\langle\Phi\rangle L^{\dagger}=\langle\Phi\rangle .
$$


Thus the Higgs vacuum expectation value breaks the global symmetry in the pattern

$$
S U(2)_{L} \times S U(2)_{R} \rightarrow S U(2)_{L+R}
$$

This is called "custodial symmetry;" actually, some authors refer to $S U(2)_{R}$ by this name [6], while others reserve it for $S U(2)_{L+R}$ [7].

Since $S U(2)$ is a three-dimensional group, the number of broken generators is $3+3-3=3$. These give rise to three massless Goldstone bosons, which are then eaten by the Higgs mechanism to provide the mass of the $W^{+}, W^{-}$, and $Z$ bosons,

$$
\begin{aligned}
M_{W}^{2} & =\frac{1}{4} g^{2} v^{2} \\
M_{Z}^{2} & =\frac{1}{4}\left(g^{2}+g^{\prime 2}\right) v^{2} .
\end{aligned}
$$

Thus

$$
\frac{M_{W}^{2}}{M_{Z}^{2}}=\frac{g^{2}}{g^{2}+g^{\prime 2}}=\cos ^{2} \theta_{W}
$$

or

$$
\rho=\frac{M_{W}^{2}}{M_{Z}^{2} \cos ^{2} \theta_{W}}=1
$$

at tree level.

Exercise 3.3 - Show that $W_{\mu}^{A}$ transforms as a triplet under global $S U(2)_{L}$ and a singlet under $S U(2)_{R}$, and hence as a triplet under the unbroken $S U(2)_{L+R}$.

Thus, in the limit $g^{\prime} \rightarrow 0, W^{+}, W^{-}, Z$ form a triplet of an unbroken global symmetry. This explains why $M_{W}=M_{Z}$ in the $g^{\prime} \rightarrow 0$ limit.

Custodial symmetry also helps us understand properties of the theory beyond tree level. Due to the unbroken $S U(2)_{L+R}$ in the $g^{\prime} \rightarrow 0$ limit, radiative corrections to the $\rho$ parameter in Eq. (66) due to gauge and Higgs bosons must be proportional to $g^{\prime 2}$. For example, the leading correction to the $\rho$ parameter from loops of Higgs bosons (Fig. 2) in the $\overline{M S}$ scheme is

$$
\hat{\rho} \approx 1-\frac{11 G_{F} M_{Z}^{2} \sin ^{2} \theta_{W}}{24 \sqrt{2} \pi^{2}} \ln \frac{m_{h}^{2}}{M_{Z}^{2}} .
$$

This correction vanishes in the limit $g^{\prime} \rightarrow 0\left(\sin ^{2} \theta_{W} \rightarrow 0\right)$. The custodial symmetry protects the tree-level relation $\rho=1$ from radiative corrections, and hence it's name. This leading

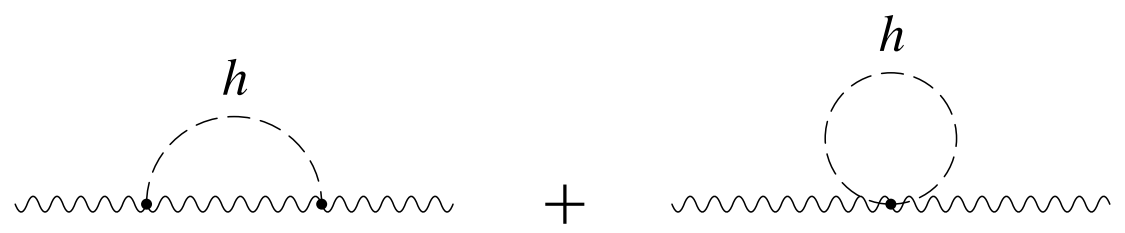

Figure 2: Virtual Higgs-boson loops contribute to the $W$ and $Z$ masses. 

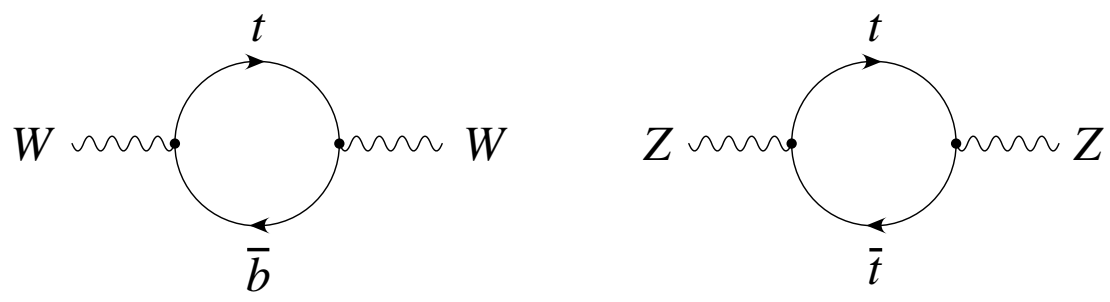

Figure 3: Virtual top-quark loops contribute to the $W$ and $Z$ masses.

correction, proportional to $\ln m_{h}$, allows us to bound the Higgs-boson mass from precision electroweak measurements.

Custodial symmetry also helps us understand radiative corrections due to massive fermions, as shown in Fig. 3. The leading correction due to loops of top and bottom quarks is [8]

$$
\hat{\rho} \approx 1+\frac{3 G_{F}}{8 \pi^{2} \sqrt{2}}\left(m_{t}^{2}+m_{b}^{2}-2 \frac{m_{t}^{2} m_{b}^{2}}{m_{t}^{2}-m_{b}^{2}} \ln \frac{m_{t}^{2}}{m_{b}^{2}}\right) .
$$

Exercise 3.4 - Show that this correction vanishes in the limit $m_{t}=m_{b}$.

Exercise 3.5 - Show that the $t, b$ Yukawa couplings have a custodial symmetry in the limit $m_{t}=m_{b}$.

This leading correction, proportional to the square of the fermion mass, allowed us to predict the top-quark mass from precision electroweak measurements before it was discovered.

Thus we see that custodial symmetry is vital to our understanding of the electroweak sector. However, the physical Higgs boson itself is not really necessary. As long as the electroweak-symmetry-breaking mechanism possesses custodial symmetry, the $\rho$ parameter equals unity at tree level and is protected from large radiative corrections.

Let's develop an effective field theory of electroweak symmetry breaking that makes custodial symmetry manifest [9]. A simple way to do this is to replace the matrix field $\Phi$ with another matrix field, $\Sigma$, which contains the Goldstone bosons, $\pi_{i}$ (which are eaten by the weak vector bosons), but does not contain a physical Higgs boson:

$$
\Phi \rightarrow \frac{v}{2} \Sigma \quad \Sigma=e^{i \frac{\sigma \cdot \pi}{v}} .
$$

The Lagrangian for electroweak symmetry breaking (EWSB) is the analogue of Eq. (151),

$$
\mathcal{L}_{E W S B}=\frac{v^{2}}{4} \operatorname{Tr}\left(D_{\mu} \Sigma\right)^{\dagger} D^{\mu} \Sigma .
$$

The Goldstone-boson matrix field $\Sigma$ transforms under custodial symmetry as

$$
\begin{aligned}
S U(2)_{L}: & \Sigma \rightarrow L \Sigma \\
S U(2)_{R}: & \Sigma \rightarrow \Sigma R^{\dagger} \\
S U(2)_{L+R}: & \Sigma \rightarrow L \Sigma L^{\dagger} .
\end{aligned}
$$


Exercise 3.6 - Let $L=e^{\frac{i}{2} \sigma \cdot \theta}$ be an $S U(2)_{L}$ transformation. Show that under an infinitesimal $S U(2)_{L}$ transformation, $\pi_{i}$ transforms non-linearly,

$$
\pi_{i} \rightarrow \frac{v}{2} \theta_{i}+\pi_{i}
$$

while under an infinitesimal $S U(2)_{L+R}$ transformation it transforms linearly,

$$
\pi_{i} \rightarrow \pi_{i}-\epsilon_{i j k} \theta_{j} \pi_{k}
$$

Since $S U(2)_{L} \times S U(2)_{R}$ is realized non-linearly, this model is called the non-linear sigma model. The fact that the symmetry is realized non-linearly means that it is broken.

Unitary gauge corresponds to $\Sigma=1$. The electroweak-symmetry-breaking Lagrangian, Eq. (170), yields the correct vector boson masses of Eq. (64). The only other dimension-two operator allowed by the $S U(2)_{L} \times U(1)_{Y}$ gauge symmetry is

$$
\mathcal{L}=c \frac{v^{2}}{4}\left[\operatorname{Tr} \sigma_{3} \Sigma^{\dagger} D_{\mu} \Sigma\right]^{2}
$$

This term contributes to the $W$ and $Z$ masses such that

$$
\rho=1+2 c
$$

Exercise 3.7 - Show that this operator violates the custodial symmetry.

This proves that custodial symmetry is sufficient to produce $\rho=1$ at leading order in the effective theory of electroweak symmetry breaking.

Beyond leading order, the effective theory has a tower of higher-dimension operators,

$$
\mathcal{L}=\frac{v^{2}}{4} \operatorname{Tr}\left(D_{\mu} \Sigma\right)^{\dagger} D^{\mu} \Sigma+\operatorname{dim} 4+\cdots
$$

The power counting is different from that of the effective field theory we previously encountered in Eq. (39), where the leading term, $\mathcal{L}_{S M}$, is renormalizable. Here the leading term, of dimension two, is non-renormalizable. The effects of the higher-dimension operators are suppressed by $E^{2} / \Lambda^{2}$, where $\Lambda \leq 4 \pi v$.

All we are really sure of is that the electroweak symmetry is broken and that the electroweak symmetry breaking sector has an approximate custodial symmetry. We don't really know if a Higgs boson exists. The Fermilab Tevatron and the CERN Large Hadron Collider will settle that question. 


\section{Scale symmetry}

Although we do not really know the mechanism of electroweak symmetry breaking, let alone whether a Higgs boson exists, it is appropriate to consider the minimal model with a single Higgs doublet as the "standard model." This is due to the remarkable fact that precision electroweak measurements are consistent with a relatively light Higgs boson, $m_{h}=114_{-45}^{+69}$ $\mathrm{GeV}$ [10]. It could have turned out that these measurements were not consistent with a Higgs boson of any mass, but that is not the way things have unfolded.

As discussed in Section 2, it is likely that the standard model is the leading term in an effective Lagrangian, Eq. (39), corresponding to an expansion in inverse powers of some large scale $M$. There are at least three hints that the scale $M$ is very large, much greater than the weak scale, $v$ :

- As discussed in Section 2, Majorana neutrino masses are of order $v^{2} / M$, which implies $M \sim 10^{14}-10^{16} \mathrm{GeV}$.

- Attempts at grand unification indicate that unification of the gauge couplings of the standard model occurs at around $10^{16} \mathrm{GeV}$.

- Quantum gravity becomes important at or before the Planck scale, $M_{P l}=\left(\hbar c / G_{N}\right)^{1 / 2} \sim$ $10^{19} \mathrm{GeV}$.

This raises a puzzle: if the fundamental scale of physics, $M$, is so high, why does the standard model reside at the ordinary energies that we observe, rather than at $M$ ? The standard model explains this in part, but not entirely.

Let's begin with fermions. As we discussed in Section 2, the fermions transform under a complex representation of the gauge symmetry. There are no fermions that are gauge singlets or transform under a real representation, so Majorana masses are forbidden. There are no pairs of fermions which transform under complex-conjugate representations of the gauge symmetry, so Dirac masses are forbidden. Thus fermions are massless until the $S U(2)_{L} \times$ $U(1)_{Y}$ symmetry is broken. Hence fermion masses are naturally of $\mathcal{O}(v)$.

Although this is a successful explanation of why fermions are light compared with $M$, it is not entirely satisfactory. Only the top quark has a mass of $\mathcal{O}(v)$; all the other charged fermions are considerably lighter. In the standard model, this is due to the very small Yukawa couplings of the fermions. It is puzzling that these couplings are so small; for the electron, the Yukawa coupling is about $10^{-5}$. These small couplings suggest that there is an approximate flavor symmetry at work (see Section 2), only weakly violated by the Yukawa couplings.

As discussed in Section 2, neutrino masses are zero at leading order in the effective theory due to an accidental lepton number symmetry. This symmetry is violated by the dimension 5 operator in Eq. (40), giving rise to a small neutrino mass of $\mathcal{O}\left(v^{2} / M\right)$. As mentioned above, this is one of the reasons we believe that $M \gg v$.

In addition to the fermion masses, the flavor sector also contains the CKM and MNS mixing matrices, whose mixing angles are listed in Table 4. The standard model accommodates, but does not explain, the pattern of masses and mixings. Hidden in this pattern are clues to physics beyond the standard model. 


\begin{tabular}{|c||c|c|}
\hline angle & quark & lepton \\
\hline$\theta_{12}$ & $13^{\circ}$ & $34^{\circ}$ \\
$\theta_{23}$ & $2.3^{\circ}$ & $45^{\circ}$ \\
$\theta_{13}$ & $0.23^{\circ}$ & $\leq 12^{\circ}$ \\
$\delta$ & $60^{\circ}$ & unknown \\
\hline \hline
\end{tabular}

Table 4: The observed mixing angles in the quark and lepton sectors. If neutrinos are Majorana, there are three unknown $C P$-violating phases; if Dirac, only one.

Let's now turn to the gauge bosons. Gauge bosons are associated with a local (gauge) symmetry that protects their masslessness. Under a gauge transformation, the gauge bosons transform as

$$
T \cdot A_{\mu} \rightarrow U T \cdot A_{\mu} U^{-1}+\frac{i}{g}\left(\partial_{\mu} U\right) U^{\dagger}
$$

where $T^{A}$ are the generators of the group. It is the second term in this transformation that forbids a mass term,

$$
\mathcal{L}=\frac{1}{2} M^{2} A_{\mu} \cdot A^{\mu}=M^{2} \operatorname{Tr} T \cdot A_{\mu} T \cdot A^{\mu}
$$

where $\operatorname{Tr} T^{A} T^{B}=\frac{1}{2} \delta^{A B}$. Thus gauge bosons are massless unless the gauge symmetry is broken. Hence the photon and gluons are massless, while the weak vector bosons naturally have a mass of $\mathcal{O}(v)$.

Gauge bosons are associated with a local symmetry, but it is not clear which way the logic runs: are they massless because of the local symmetry, or is the local symmetry present because they are massless? It's not even clear that it makes sense to think about it either of these ways. For many years physicists considered the local symmetry to be fundamental, but that position is no longer held sacred. For example, in string theory there are massless, spinone modes of the string, and at low energy these are described by an effective gauge theory, even though the underlying string theory is not based on gauge symmetry [2. Perhaps even more remarkable are supersymmetric field theories with electric-magnetic duality. There are examples in which the long-distance (magnetic) degrees of freedom include massless gauge bosons with a local symmetry, yet the short-distance (electric) degrees of freedom do not possess this local symmetry [1].

Because of its importance, I want to dwell in more detail on the connection between massless gauge bosons and gauge symmetry. For simplicity, I'll specialize to QED, although the results for a non-Abelian gauge theory are analogous.

Gauge symmetry implies a Ward identity. In the case of the photon self energy $\Pi_{\mu \nu}$, shown in Fig. 4, the Ward identity is

$$
q^{\mu} \Pi_{\mu \nu}=0
$$

where $q$ is the photon four momentum. This implies that the self energy must have the form

$$
\Pi_{\mu \nu}=\left(q^{2} g_{\mu \nu}-q_{\mu} q_{\nu}\right) \Pi\left(q^{2}\right) .
$$

We can calculate the effect of the photon self energy on the photon propagator by summing the geometric series shown in Fig. 5. It is particularly easy to do this in Landau gauge, 


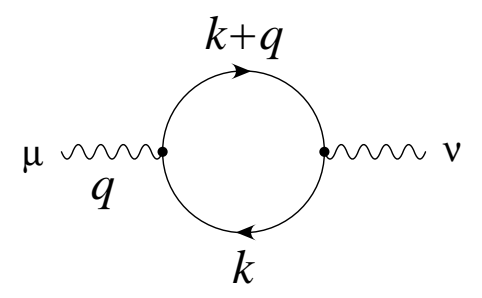

Figure 4: Photon self-energy diagram.

where the numerator of the propagator has the same form as the self energy, Eq. (79). We find

$$
\begin{aligned}
& \frac{-i}{q^{2}}\left(g^{\mu \nu}-\frac{q^{\mu} q^{\nu}}{q^{2}}\right)+\frac{-i}{q^{2}}\left(g^{\mu \rho}-\frac{q^{\mu} q^{\rho}}{q^{2}}\right) i\left(q^{2} g_{\rho \sigma}-q_{\rho} q_{\sigma}\right) \Pi \frac{-i}{q^{2}}\left(g^{\sigma \nu}-\frac{q^{\sigma} q^{\nu}}{q^{2}}\right)+\cdots \\
& =\frac{-i}{q^{2}}\left(g^{\mu \nu}-\frac{q^{\mu} q^{\nu}}{q^{2}}\right)[1+\Pi+\cdots] \\
& =\frac{-i}{q^{2}[1-\Pi]}\left(g^{\mu \nu}-\frac{q^{\mu} q^{\nu}}{q^{2}}\right) .
\end{aligned}
$$

Thus the propagator acquires a factor $[1-\Pi]^{-1}$.

Let's now proceed to calculate the contribution to $\Pi$ from a fermion loop. Using the Feynman diagram in Fig. 4, we find [1]

$$
i \Pi_{\mu \nu}=i\left(q^{2} g_{\mu \nu}-q_{\mu} q_{\nu}\right) \Pi=(-i e Q)^{2} i^{2}(-1) \int \frac{d^{N} k}{(2 \pi)^{N}} \frac{\operatorname{Tr} \gamma_{\mu}(\not k+m) \gamma_{\nu}(\not k+\not k+m)}{\left(k^{2}-m^{2}\right)\left((k+q)^{2}-m^{2}\right)}
$$

where $Q$ is the fermion electric charge and the factor $(-1)$ is from the fermion loop. Let's calculate in $N$ dimensions, in order to regulate the ultraviolet divergence. Contracting both sides of this equation with $g^{\mu \nu}$ yields

$$
i\left(q^{2} N-q^{2}\right) \Pi=-e^{2} Q^{2} \int \frac{d^{N} k}{(2 \pi)^{N}} \frac{\operatorname{Tr} \gamma_{\mu}(\not k+m) \gamma^{\mu}(\not k+\not q+m)}{\left(k^{2}-m^{2}\right)\left((k+q)^{2}-m^{2}\right)}
$$

where I've used $g^{\mu \nu} g_{\mu \nu}=N$. The trace gives

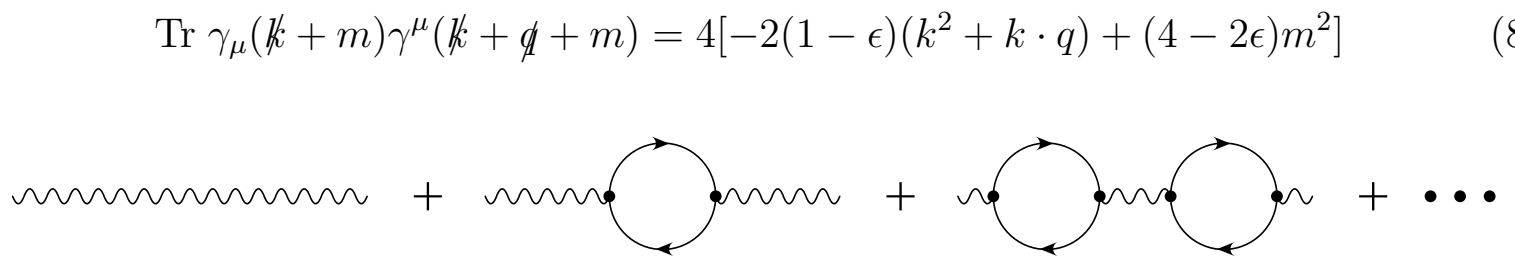

Figure 5: The renormalized photon propagator from a sum of self-energy diagrams. 
where I've used

$$
\begin{aligned}
\gamma_{\mu} \gamma^{\mu} & =N \equiv 4-2 \epsilon \\
\gamma_{\mu} \phi \gamma^{\mu} & =-2(1-\epsilon) \not l .
\end{aligned}
$$

Now let's rewrite the numerator in terms of factors appearing in the denominator,

$$
k^{2}+k \cdot q=\frac{1}{2}\left[(k+q)^{2}-m^{2}+\left(k^{2}-m^{2}\right)-q^{2}+2 m^{2}\right] .
$$

This allows us to write Eq. (82) in the simple form

$$
\begin{aligned}
& i(3-2 \epsilon) q^{2} \Pi=-4 e^{2} Q^{2} \\
& \int \frac{d^{N} k}{(2 \pi)^{N}}\left[(\epsilon-1)\left(\frac{1}{\left(k^{2}-m^{2}\right)}+\frac{1}{\left((k+q)^{2}-m^{2}\right)}\right)+\frac{(1-\epsilon) q^{2}+2 m^{2}}{\left(k^{2}-m^{2}\right)\left((k+q)^{2}-m^{2}\right)}\right]
\end{aligned}
$$

Let's first work in $N=4$ dimensions $(\epsilon=0)$. The first two integrals above are quadratically divergent. Let's simply cut them off. The first integral gives

$$
\int \frac{d^{4} k}{(2 \pi)^{4}} \frac{1}{\left(k^{2}-m^{2}\right)}=i \int \frac{d^{4} k_{E}}{(2 \pi)^{4}} \frac{1}{\left(-k_{E}^{2}-m^{2}\right)} \sim-\frac{i}{16 \pi^{2}} M^{2}
$$

where I've Wick rotated to Euclidean space $\left(k^{0} \rightarrow i k_{E}^{0}\right)$ and used $d^{4} k_{E}=\pi^{2} k_{E}^{2} d k_{E}^{2}$ (after angular integration) to evaluate the integral, cut off at $k_{E}^{2}=M^{2}$. The second integral gives the same result after first performing the shift $k \rightarrow k-q$. Thus we find

$$
\Pi \sim-\frac{e^{2}}{6 \pi^{2}} Q^{2} \frac{M^{2}}{q^{2}} .
$$

Inserting this into Eq. (80), we see that the photon has acquired a (tachyonic) mass of $\mathcal{O}(M)$ :

$$
\frac{-i}{q^{2}[1-\Pi]} \sim \frac{-i}{q^{2}+\frac{e^{2}}{6 \pi^{2}} Q^{2} M^{2}} .
$$

What went wrong? Although it is not evident from our calculation, simply cutting off the integral violates the Ward identity, Eq. (78) [1]:

$$
\Pi_{\mu \nu} \sim M^{2} g_{\mu \nu} \rightarrow q^{\mu} \Pi_{\mu \nu} \neq 0
$$

Thus the Ward identity, which follows from gauge symmetry, is essential to protect the masslessness of the photon.

Rather than using a cutoff, we can evaluate the photon self energy in $N$ dimensions, which respects the Ward identity. The quadratically divergent integrals give

$$
\int \frac{d^{N} k}{(2 \pi)^{N}} \frac{1}{\left(k^{2}-m^{2}\right)}=\int \frac{d^{N} k}{(2 \pi)^{N}} \frac{1}{\left((k+q)^{2}-m^{2}\right)}=-\frac{i}{(4 \pi)^{N / 2}} \Gamma(\epsilon-1)\left(m^{2}\right)^{1-\epsilon} .
$$

The pole at $\epsilon=1(N=2)$ signals the quadratic divergence. However, these integrals are multiplied by a factor $(\epsilon-1)$ in Eq. (85). Using

$$
(\epsilon-1) \Gamma(\epsilon-1)=\Gamma(\epsilon)
$$


we see that the pole at $\epsilon=1$, and hence the quadratic divergence, was illusory. The quadratic dependence on the cutoff $M$ that we discovered above was an artifact of using a regulator that violates the Ward identity.

After some additional work, one finds [1]

$$
\Pi=-\frac{8 e^{2} Q^{2}}{(4 \pi)^{N / 2}} \Gamma(\epsilon) \int_{0}^{1} d x \frac{x(1-x)}{\left[m^{2}-x(1-x) q^{2}\right]^{\epsilon}} .
$$

The pole at $\epsilon=0(N=4)$ signals a logarithmic divergence. Inserting this into Eq. (80), we find that the photon propagator still has a pole at $q^{2}=0$, so the photon remains massless, thanks to the Ward identity.

Thus the standard model successfully explains why fermions and gauge bosons are so much lighter than the hypothesized fundamental scale, $M$. Now let's turn to scalars. Here is where the standard model is not so successful. A scalar mass term is of the form

$$
\mathcal{L}=-m^{2} \phi^{\dagger} \phi
$$

and this is always allowed regardless of the gauge symmetry. Thus there is no reason for scalars to be light compared to $M$. In the standard model, this would mean that the Higgs field would naturally have a mass of $\mathcal{O}(M)$, and thus not be available at low energy to break the electroweak symmetry.

There are at least two ways to avoid this conclusion. One way is to make the scalars Goldstone bosons of some broken global symmetry. As we saw in Exercise 3.6, Goldstone bosons transform as $\pi_{i} \rightarrow \frac{v}{2} \theta_{i}+\pi_{i}$, which forbids a mass term, $\mathcal{L}=-m^{2} \pi_{i} \pi_{i}$. Perhaps the Higgs field is a (pseudo)-Goldstone boson of some (approximate) broken global symmetry. This is the idea behind the currently popular "little Higgs" models [12].

The other generic way to make scalars massless is to use supersymmetry [13. A scalar field is replaced by a scalar superfield, $\phi \rightarrow \Phi$ (not to be confused with the matrix field $\Phi$ of Section 3). The superpotential $W(\Phi)$ depends only on $\Phi$, not $\Phi^{*}$, which is referred to as the property of holomorphy. If the scalar field transforms under a complex or pseudoreal representation of some global or local group, then a mass term, $W(\Phi)=\frac{1}{2} m \Phi^{2}$, is forbidden, exactly as we saw for fermions in Section 2 . Since supersymmetry connects bosons and fermions, it is not surprising that the same method we used to make fermions light can also be used to make scalars light.

Unfortunately, the minimal supersymmetric standard model does not make use of this method. That model requires two light Higgs-doublet superfields, $H_{1}$ and $H_{2}$, with opposite hypercharge, in order to provide masses to both up-type and down-type quarks. Thus a mass term in the superpotential

$$
W\left(H_{1}, H_{2}\right)=\mu H_{2}^{T} \epsilon H_{1}
$$

is allowed by the gauge symmetry. However, this does not mean that supersymmetry is irrelevant to understanding why the Higgs scalars are light compared to $M$, as we will discuss shortly.

We conclude that in the standard model, there is no principle to tell us why the Higgs mass is light compared to the hypothesized scale of fundamental physics, $M$. It is not 


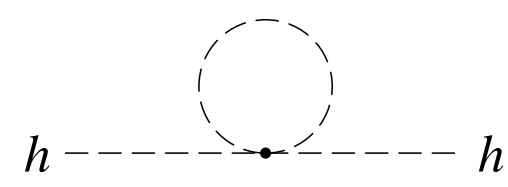

Figure 6: Quadratically divergent contribution to the Higgs-boson mass.

unreasonable to regard this as a merely "aesthetic" problem. However, there is an even worse problem: it is not natural for a scalar mass to be much lighter than $M$. Consider the one-loop contribution to the scalar mass shown in Fig. 6. This diagram has a genuine quadratic divergence, so the relation between the Higgs mass evaluated at low energy and at the scale $M$ is

$$
m^{2}(m)=m^{2}(M)+\frac{3}{4 \pi^{2}} \lambda M^{2}
$$

where $\lambda$ is the Higgs-field self coupling, Eq. (46). In order to obtain $m^{2}(0) \ll M^{2}$, two things must happen:

- $m^{2}(M) \sim M^{2}$ - this is perfectly natural

- $\frac{3}{4 \pi^{2}} \lambda M^{2}$ must cancel $m^{2}(M)$ to high accuracy. There is no principle to enforce this.

This is the famous "hierarchy problem."

A supersymmetric theory has no quadratic divergences, so it solves this "technical" aspect of the hierarchy problem. Instead, one is left with the "aesthetic" problem we already encountered above. Fortunately, there are dynamical ways to solve this, so this is a much softer version of the hierarchy problem.

To summarize, we have argued that the standard model successfully explains why fermion and gauge boson masses are of $\mathcal{O}(v)$, and hence much lighter than the hypothesized scale of fundamental physics, $M$. However, the standard model fails to explain why the Higgs mass, or equivalently $v$, is much lighter than $M$ in the first place. This "hierarchy problem" has occupied theorists for a long time, and there are many potential solutions, including:

- Perhaps $M$ is actually not much larger than $v$, so there is no hierarchy after all. Theories with extra dimensions are a recent attempt along this direction.

- Perhaps $M$ is only a little bigger than $v$, so it's really only a "little hierarchy." This is the rationale behind "little Higgs" models [12].

- Perhaps $M$ really is much larger than $v$, and low-energy supersymmetry makes this natural [13].

- Perhaps there are no fundamental scalars in nature after all, and the electroweak symmetry is broken some other way. For example, Technicolor models break the electroweak symmetry via a fermion-antifermion condensate [14. 
If there really are no light fundamental scalars in nature, we have an explanation at the ready. Perhaps the hierarchy problem is not a problem at all, but just a successful explanation of why there are no light fundamental scalars.

No amount of deliberation by theorists alone is going to decide which, if any, of these solutions is correct. Experiment will decide. The Fermilab Tevatron is already probing the physics of electroweak symmetry breaking, and the CERN Large Hadron Collider is sure to give us a lot of information. Our goal is to unravel the mechanism of electroweak symmetry breaking, perhaps with the assistance of a Linear $\left(e^{+} e^{-}\right)$Collider. It is going to take a lot of effort on the part of both theorists and experimentalists, and it is going to be an awful lot of fun.

\section{Acknowledgments}

I would like to thank John Terning, Carlos Wagner, and Dieter Zeppenfeld for the opportunity to present these lectures, and the TASI students for their questions and comments. I am grateful for assistance from A. de Gouvea, B. Kayser, R. Leigh, T. McElmurry, J. Sayre, and Sören Wiesenfeldt. This work was supported in part by the U. S. Department of Energy under contract No. DE-FG02-91ER40677.

\section{Solutions to the exercises}

\section{Section 1}

Exercise 1.1 - For example,

$$
\begin{aligned}
{\left[A_{i}, A_{j}\right] } & =\frac{1}{4}\left(\left[J_{i}, J_{j}\right]+i\left[J_{i}, K_{j}\right]+i\left[K_{i}, J_{j}\right]-\left[K_{i}, K_{j}\right]\right) \\
& =\frac{1}{4} i \epsilon_{i j k}\left(J_{k}+i K_{k}+i K_{k}+J_{k}\right) \\
& =i \epsilon_{i j k} A_{k}
\end{aligned}
$$

and similarly for the other commutators.

Exercise 1.2 - The (0,1/2) representation is given by

$$
\begin{aligned}
A_{i} & =0 \\
B_{i} & =\frac{1}{2} \sigma_{i}
\end{aligned}
$$

which corresponds to

$$
\begin{aligned}
J_{i} & =\frac{1}{2} \sigma_{i} \\
i K_{i} & =-\frac{1}{2} \sigma_{i} .
\end{aligned}
$$


Hence the $(0,1 / 2)$ representation transforms under rotations and boosts as

$$
\begin{aligned}
& \chi \rightarrow e^{-\frac{i}{2} \sigma \cdot \theta} \chi \\
& \chi \rightarrow e^{\frac{1}{2} \sigma \cdot \eta} \chi .
\end{aligned}
$$

Only the boost transformation differs from that of the $(1 / 2,0)$ representation, Eq. (11).

Exercise 1.3 - Gluinos are the superpartners of gluons, and therefore transform under the adjoint representation, which is real.

Exercise 1.4 - Under an $S U(2)$ transformation,

$$
\epsilon_{a b} \chi^{a T} \epsilon \chi^{b} \rightarrow \epsilon_{a b} U_{c}^{a} \chi^{c T} \epsilon U_{d}^{b} \chi^{d} \rightarrow \epsilon_{c d} \chi^{c T} \epsilon \chi^{d}
$$

where I've used $\epsilon_{a b} U_{c}^{a} U_{d}^{b}=\operatorname{det} U \epsilon_{c d}=\epsilon_{c d}$. However,

$$
\left(\chi^{a T} \epsilon \chi^{b}\right)^{T}=\chi^{b T} \epsilon \chi^{a}
$$

where I've used the fact that the spinor fields are Grassmann variables. Hence $\chi^{a T} \epsilon \chi^{b}$ is symmetric under interchange of $a, b$, and vanishes when contracted with $\epsilon_{a b}$.

This exercise demonstrates a more general result: a Majorana mass term cannot be constructed for a Weyl spinor that transforms under a pseudoreal representation of a global or local symmetry, because the tensor used to construct the mass term (the analogue of $\left.\epsilon_{a b}\right)$ is necessarily antisymmetric [1. A pseudoreal representation is one that is unitarily equivalent to its complex conjugate: $U^{*}=S U S^{\dagger}$ for some unitary transformation $S$. For $S U(2), S=\epsilon$.

Exercise 1.5 - Under rotations,

$$
\epsilon \xi^{*} \rightarrow \epsilon M^{*} \xi^{*}=M \epsilon \xi^{*}
$$

where I have used the fact that $M=e^{-\frac{i}{2} \sigma \cdot \theta}$ is unitary if it is a rotation. Under boosts,

$$
\epsilon \xi^{*} \rightarrow \epsilon M^{*} \xi^{*}=M^{-1} \epsilon \xi^{*}
$$

where I have used the fact that $M=e^{-\frac{1}{2} \sigma \cdot \eta}$ is Hermitian if it is a boost. This shows that $\epsilon \xi^{*}$ transforms under the $(0,1 / 2)$ representation of the Lorentz group (see Exercise 1.2).

Exercise 1.6 - Eq. (15) may be rewritten

$$
\begin{aligned}
-\frac{1}{2} m \bar{\psi}_{M} \psi_{M} & =-\frac{1}{2} m\left(\chi^{\dagger},-\chi^{T} \epsilon\right)\left(\begin{array}{ll}
0 & 1 \\
1 & 0
\end{array}\right)\left(\begin{array}{c}
\chi \\
\epsilon \chi^{*}
\end{array}\right) \\
& =\frac{1}{2} m\left(\chi^{T} \epsilon \chi-\chi^{\dagger} \epsilon \chi^{*}\right)
\end{aligned}
$$

which is equivalent to Eq. (21).

Exercise 1.7 - Eq. (17) may be rewritten

$$
\begin{aligned}
-m\left(\left(\psi^{c}\right)_{L}^{T} C \psi_{L}+\text { h.c. }\right) & =-m\left(\left(\xi^{T}, 0\right)\left(\begin{array}{cc}
-\epsilon & 0 \\
0 & \epsilon
\end{array}\right)\left(\begin{array}{c}
\chi \\
0
\end{array}\right)+\text { h.c. }\right) \\
& =m\left(\xi^{T} \epsilon \chi+\text { h.c. }\right)
\end{aligned}
$$


which is Eq. (9).

Exercise 1.8 - Eq. (18) may be rewritten

$$
\begin{aligned}
-\frac{1}{2} m\left(\psi_{L}^{T} C \psi_{L}+\text { h.c. }\right) & =-\frac{1}{2} m\left(\left(\chi^{T}, 0\right)\left(\begin{array}{cc}
-\epsilon & 0 \\
0 & \epsilon
\end{array}\right)\left(\begin{array}{c}
\chi \\
0
\end{array}\right)+\text { h.c. }\right) \\
& =\frac{1}{2} m\left(\chi^{T} \epsilon \chi+\text { h.c. }\right)
\end{aligned}
$$

which is Eq. (2).

Exercise 1.9 - Using the definition of a conjugate spinor, we find

$$
\begin{aligned}
\psi_{M}^{c} & \equiv C \gamma^{0} \psi_{M}^{*} \\
& =\left(\begin{array}{cc}
-\epsilon & 0 \\
0 & \epsilon
\end{array}\right)\left(\begin{array}{ll}
0 & 1 \\
1 & 0
\end{array}\right)\left(\begin{array}{c}
\chi^{*} \\
\epsilon \chi
\end{array}\right) \\
& =\left(\begin{array}{c}
\chi \\
\epsilon \chi^{*}
\end{array}\right)=\psi_{M} .
\end{aligned}
$$

Exercise 1.10 - Eq. (23) may be rewritten

$$
\begin{aligned}
-\frac{1}{2} m\left({\overline{\left(\psi^{c}\right)}}_{R} \psi_{L}+\text { h.c. }\right) & =-\frac{1}{2} m\left(\left(0,-\chi^{T} \epsilon\right)\left(\begin{array}{ll}
0 & 1 \\
1 & 0
\end{array}\right)\left(\begin{array}{c}
\chi \\
0
\end{array}\right)+\text { h.c. }\right) \\
& =\frac{1}{2} m\left(\chi^{T} \epsilon \chi+\text { h.c. }\right)
\end{aligned}
$$

which is Eq. (2).

\section{Section 2}

Exercise 2.1 - Under an $S U(2)_{L}$ transformation,

$$
\epsilon \phi^{*} \rightarrow \epsilon U^{*} \phi^{*}=U \epsilon \phi^{*}
$$

using the relation $\epsilon U^{*}=U \epsilon$ derived in Exercise 1.5 (in that case $U=M$ was a spatial rotation, but the group is still $S U(2))$.

Exercise 2.2 - The lepton sector has one complex Yukawa matrix $\Gamma_{e}^{i j}$, and two $U(3)$ symmetries of the matter Lagrangian, minus lepton number which is a symmetry of the full Lagrangian, and cannot be used to diagonalize the mass matrix. Thus the number of parameters is

$$
2 \times 3 \times 3-(3 \times 3 \times 2-1)=1 .
$$

However, we know that $m_{e}, m_{\mu}, m_{\tau}$ are independent, so there are actually 3 parameters. Thus there must be three symmetries of the full Lagrangian that we need to subtract from the $U(3)$ symmetries, not just one. These are the individual lepton numbers $L_{e}, L_{\mu}, L_{\tau}$ (where $\left.L=L_{e}+L_{\mu}+L_{\tau}\right)$. 
Exercise 2.3 - $L_{L}$ and $\phi$ have equal and opposite hypercharge, so $N_{R}$ must have zero hypercharge in order for $\mathcal{L}_{\text {Yukawa }}$ to be gauge invariant. Similarly, $L_{L}$ and $\epsilon \phi^{*}$ both transform as $S U(2)_{L}$ doublets, so $\mathcal{L}_{\text {Yukawa }}$ is gauge invariant if $N_{R}$ is inert under $S U(2)_{L}$. In order for $\mathcal{L}_{Y \text { ukawa }}$ to conserve lepton number, we must assign $L=+1$ to $N_{R}$.

Exercise 2.4 - The combination $L_{L}^{T} \epsilon \phi$ is $S U(2)_{L} \times U(1)_{Y}$ invariant (see Exercise 2.3), so $\mathcal{L}_{5}$ is gauge invariant. Lepton number is violated because $L_{L}$ carries $L=+1$, so $\mathcal{L}_{5}$ has $L=+2$.

Exercise 2.5 - The counting is similar to that of Exercise 2.2, with two differences. First, we must add the complex, symmetric matrix $c^{i j}$, which has $2 \times 6$ parameters. Second, we should not subtract lepton number from the symmetries of the matter Lagrangian, because it is no longer a symmetry of the full Lagrangian, being violated by $\mathcal{L}_{5}$. Hence the number of physically relevant parameters is

$$
2 \times 3 \times 3+2 \times 6-3 \times 3 \times 2=12
$$

Of these parameters, six are the charged-lepton and neutrino masses, leaving six parameters for the MNS matrix. Three are mixing angles, and three are $C P$-violating phases.

Exercise 2.6 - It's easiest to do this with index-free notation:

$$
\frac{\partial \mathcal{L}}{\partial N_{R}}=-\bar{L}_{L} \Gamma_{\nu} \epsilon \phi^{*}-N_{R}^{T} M_{R} C+\text { h.c. . }
$$

Solving for $N_{R}$ gives

$$
N_{R}=\phi^{\dagger} \epsilon C \gamma^{0}\left(\Gamma_{\nu} M_{R}^{-1}\right)^{T} L_{L}^{*}
$$

Plugging this back into $\mathcal{L}$ to eliminate $N_{R}$ gives

$$
\mathcal{L}=\frac{1}{2} L_{L}^{\dagger} \epsilon \phi^{*} C \Gamma_{\nu}\left(\Gamma_{\nu} M_{R}^{-1}\right)^{T} \phi^{\dagger} \epsilon L^{*}+\text { h.c. } .
$$

This is equal to Eq. (40), where we identify the first term above with the h.c. term of $\mathcal{L}_{5}$. This gives

$$
\frac{c^{\dagger}}{M}=-\frac{1}{2} \Gamma_{\nu}\left(\Gamma_{\nu} M_{R}^{-1}\right)^{T}
$$

which is equivalent to Eq. (44) if we recall that $c$ is a symmetric matrix.

\section{Section 3}

Exercise 3.1 - Writing a matrix as an outer-product of two vectors,

$$
\operatorname{Tr} \Phi^{\dagger} \Phi=\frac{1}{2} \operatorname{Tr}\left(\begin{array}{c}
-\phi^{T} \epsilon \\
\phi^{\dagger}
\end{array}\right)\left(\epsilon \phi^{*}, \phi\right)=\phi^{\dagger} \phi
$$

where I've used $\phi^{T} \phi^{*}=\left(\phi^{T} \phi^{*}\right)^{T}=\phi^{\dagger} \phi$. This shows that the potentials are equivalent. For the gauge-covariant kinetic-energy term, we need

$$
D_{\mu} \Phi=\left(D_{\mu}\left(\epsilon \phi^{*}\right), D_{\mu} \phi\right)=\left(\epsilon\left(D_{\mu} \phi\right)^{*}, D_{\mu} \phi\right)
$$


where the last step uses $\sigma \epsilon=-\epsilon \sigma^{T}=-\epsilon \sigma^{*}$. From there the proof proceeds as above.

Exercise 3.2 - Consider the $U(1)$ subgroup of $S U(2)_{R}$ given by $e^{\frac{i}{2} \sigma_{3} \theta}$. Inserting this for the transformation $R$ in Eq. (157) yields the hypercharge transformation of Eq. (54).

Exercise $3.3-W_{\mu}^{A}$ transforms under an $S U(2)_{L}$ gauge transformation as

$$
\frac{\sigma}{2} \cdot W_{\mu} \rightarrow L \frac{\sigma}{2} \cdot W_{\mu} L^{\dagger}+\frac{i}{g}\left(\partial_{\mu} L\right) L^{\dagger}
$$

Under a global $S U(2)_{L}$ transformation, the second term is absent, and $W_{\mu}^{A}$ transforms simply under the adjoint (triplet) representation of $S U(2)_{L}$. Since $W_{\mu}^{A}$ is a singlet under $S U(2)_{R}$, it also transforms as a triplet under $S U(2)_{L+R}$.

Exercise 3.4 - Using l'Hôpital's rule for sick functions gives

$$
\lim _{x \rightarrow 1} \frac{\ln x}{x-1}=\lim _{x \rightarrow 1} \frac{1}{x}=1
$$

where $x=m_{t}^{2} / m_{b}^{2}$. Thus the correction, which is proportional to

$$
x+1-2 \frac{x}{x-1} \ln x
$$

vanishes in the limit $x \rightarrow 1$.

Exercise 3.5 - In the limit $m_{t}=m_{b}$, the top and bottom Yukawa couplings are equal, and the Yukawa Lagrangian of Eq. (29) (restricted to the third generation of quarks) may be written

$$
\mathcal{L}_{\text {Yukawa }}=-y \bar{Q}_{L} \Phi Q_{R}+\text { h.c. }
$$

where $Q_{R}=\left(t_{R}, b_{R}\right)$ is the analogue of $Q_{L}$ for the right-chiral quark fields. Under $S U(2)_{L}$, $Q_{L} \rightarrow L Q_{L}$. If we let $Q_{R}$ transform under $S U(2)_{R}$ as $Q_{R} \rightarrow R Q_{R}$, then the Yukawa Lagrangian is invariant under $S U(2)_{L} \times S U(2)_{R}$ custodial symmetry:

$$
\bar{Q}_{L} \Phi Q_{R} \rightarrow \bar{Q}_{L} L^{\dagger} L \Phi R^{\dagger} R Q_{R}=\bar{Q}_{L} \Phi Q_{R}
$$

Exercise 3.6 - Under an infinitesimal $S U(2)_{L}$ transformation,

$$
\begin{aligned}
e^{i \frac{\sigma \cdot \pi}{v}} & \rightarrow e^{\frac{i}{2} \sigma \cdot \theta} e^{i \frac{\sigma \cdot \pi}{v}} \\
1+i \frac{\sigma \cdot \pi}{v}+\cdots & \rightarrow\left(1+\frac{i}{2} \sigma \cdot \theta+\cdots\right)\left(1+i \frac{\sigma \cdot \pi}{v}+\cdots\right) \\
\pi_{i} & \rightarrow \frac{v}{2} \theta_{i}+\pi_{i}
\end{aligned}
$$

while under an infinitesimal $S U(2)_{L+R}$ transformation

$$
\begin{aligned}
e^{i \frac{\sigma \cdot \pi}{v}} & \rightarrow e^{\frac{i}{2} \sigma \cdot \theta} e^{i \frac{\sigma \cdot \pi}{v}} e^{-\frac{i}{2} \sigma \cdot \theta} \\
1+i \frac{\sigma \cdot \pi}{v}+\cdots & \rightarrow\left(1+\frac{i}{2} \sigma \cdot \theta+\cdots\right)\left(1+i \frac{\sigma \cdot \pi}{v}+\cdots\right)\left(1-\frac{i}{2} \sigma \cdot \theta+\cdots\right) \\
& =1+i \frac{\sigma \cdot \pi}{v}-\frac{1}{2 v} \theta_{i} \pi_{j}\left[\sigma_{i}, \sigma_{j}\right]+\cdots \\
\pi_{i} & \rightarrow \pi_{i}-\epsilon_{i j k} \theta_{j} \pi_{k}
\end{aligned}
$$


where I've used $\left[\sigma_{i}, \sigma_{j}\right]=2 i \epsilon_{i j k} \sigma_{k}$.

Exercise 3.7 - The operator is invariant under global $S U(2)_{L}$ (as it must be, since it is also invariant under local $\left.S U(2)_{L}\right)$,

$$
\operatorname{Tr} \sigma_{3} \Sigma^{\dagger} D_{\mu} \Sigma \rightarrow \operatorname{Tr} \sigma_{3} \Sigma^{\dagger} L^{\dagger} D_{\mu} L \Sigma=\operatorname{Tr} \sigma_{3} \Sigma^{\dagger} D_{\mu} \Sigma
$$

but not under $S U(2)_{R}$,

$$
\operatorname{Tr} \sigma_{3} \Sigma^{\dagger} D_{\mu} \Sigma \rightarrow \operatorname{Tr} \sigma_{3} R \Sigma^{\dagger} D_{\mu} \Sigma R^{\dagger} \neq \operatorname{Tr} \sigma_{3} \Sigma^{\dagger} D_{\mu} \Sigma
$$

because of the presence of $\sigma_{3}$. Only the hypercharge subgroup of $S U(2)_{R}$ is respected (see Exercise 3.2 ), as it must be. 


\section{References}

[1] M. E. Peskin and D. V. Schroeder, An Introduction to Quantum Field Theory (AddisonWesley, Reading, 1995).

[2] S. Weinberg, The Quantum Theory of Fields, Vol. 1: Foundations (Cambridge, 1995).

[3] J. Wess and J. Bagger, Supersymmetry and Supergravity (Princeton, 1992).

[4] B. Kayser, F. Gibrat-Debu and F. Perrier, The Physics Of Massive Neutrinos, World Sci. Lect. Notes Phys. 25, 1 (1989).

[5] A. F. Falk, "The CKM matrix and the heavy quark expansion," in Flavor Physics for the Millennium, TASI 2000, ed. J. Rosner (World Scientific, Singapore, 2001), p. 379 arXiv:hep-ph/0007339.

[6] H. Georgi, "Effective field theory," Ann. Rev. Nucl. Part. Sci. 43, 209 (1993).

[7] P. Sikivie, L. Susskind, M. B. Voloshin and V. I. Zakharov, "Isospin Breaking In Technicolor Models," Nucl. Phys. B 173, 189 (1980).

[8] M. J. G. Veltman, Diagrammatica: The Path to Feynman Rules (Cambridge, 1994).

[9] R. S. Chivukula, M. J. Dugan, M. Golden and E. H. Simmons, "Theory of a strongly interacting electroweak symmetry breaking sector," Ann. Rev. Nucl. Part. Sci. 45, 255 (1995) arXiv:hep-ph/9503230.

[10] S. Eidelman et al. [Particle Data Group Collaboration], Phys. Lett. B 592, 1 (2004).

[11] J. H. Schwarz and N. Seiberg, "String theory, supersymmetry, unification, and all that," Rev. Mod. Phys. 71, S112 (1999) arXiv:hep-th/9803179.

[12] M. Schmaltz, "Physics beyond the standard model (Theory): Introducing the little Higgs," Nucl. Phys. Proc. Suppl. 117, 40 (2003) arXiv:hep-ph/0210415.

[13] S. P. Martin, "A supersymmetry primer," in Perspectives on Supersymmetry, ed. G. Kane (World Scientific, Singapore, 1998), p. 1 arXiv:hep-ph/9709356.

[14] C. T. Hill and E. H. Simmons, "Strong dynamics and electroweak symmetry breaking," Phys. Rept. 381, 235 (2003) [Erratum-ibid. 390, 553 (2004)] arXiv:hep-ph/0203079|. 